\title{
Locally compact perfectly normal spaces may all be paracompact
}

\author{
by \\ Paul B. Larson (Oxford, OH) and Franklin D. Tall (Toronto)
}

\begin{abstract}
We work towards establishing that if it is consistent that there is a supercompact cardinal then it is consistent that every locally compact perfectly normal space is paracompact. At a crucial step we use some still unpublished results announced by Todorcevic. Modulo this and the large cardinal, this answers a question of S. Watson. Modulo these same unpublished results, we also show that if it is consistent that there is a supercompact cardinal, it is consistent that every locally compact space with a hereditarily normal square is metrizable. We also solve a problem raised by the second author, proving it consistent with ZFC that every first countable hereditarily normal countable chain condition space is hereditarily separable.
\end{abstract}

0. Introduction. Only a few of the implications concerning basic properties in general topology have remained open. One raised by Watson [40 42. is particularly interesting and is characterized in [42] as his favorite problem:

Is it consistent that every locally compact perfectly normal space is paracompact?

If this implication holds, then locally compact, perfectly normal spaces have a very simple structure; they are simply the topological sum of $\sigma$-compact, perfectly normal - hence hereditarily Lindelöf and first countablespaces. In fact, as we shall see, these pieces may be taken to be hereditarily separable as well.

Continuing the theme of "niceness", let us note that many of the notorious counterexamples of set-theoretic topology are ruled out: every perfectly normal manifold is metrizable, every locally compact normal Moore space is metrizable, there are no Ostaszewski spaces and so forth. Watson [4] remarks, (...) a consistent theorem would be amazing. (...) It looks impossible

2010 Mathematics Subject Classification: Primary 54D15, 54D20, 54D45, 03E35, 54A35; Secondary 03E50, 03E65, 03E75, 54A20, 54A25.

Key words and phrases: locally compact, perfectly normal, paracompact, PFA, Souslin tree, hereditarily normal. 
to me. The reason for this hyperbole is that, at the time, no known model could embody the required combinatorics. In fact, a tantalizing aspect of the problem is that a positive solution follows from the conjunction of two statements known to follow from well-known but mutually inconsistent axioms. Specifically,

(1) $V=L$ implies locally compact perfectly normal spaces are collectionwise Hausdorff.

(2) $\mathrm{MA}+\sim \mathrm{CH}$ implies locally compact perfectly normal collectionwise Hausdorff spaces are paracompact.

We shall show that, assuming the existence of large cardinals, there is a model in which both conclusions hold, answering Watson's question. Precisely,

THEOREM 1. If it is consistent that there is a supercompact cardinal, it is consistent that every locally compact perfectly normal space is paracompact.

The key to establishing Theorem 1 is to employ a model in which wellknown topological consequences both of PFA and of $V=L$ hold. From the conjunction of such consequences, many new interesting topological results easily follow. Before stating such consequences and our new results, we need to set out some context.

Our set-theoretic notation is standard, as in [17. All $\omega_{1}$-trees are presumed to be normal, in the terminology of [14]. If $S$ is a tree and $\alpha$ is an ordinal, we let $S(\alpha)$ denote the $\alpha$ th level of $S$. Topological notation is from Engelking [8]. Since we mainly deal with locally compact spaces, it is convenient to assume all spaces are Hausdorff unless otherwise stated. However, note that the various results quoted about normality implying collectionwise Hausdorffness do not in fact require the assumption of Hausdorffness.

The context we shall consider is in the same family as that used to prove the consistency of the positive solution to Katětov's problem [21]. This approach will surely find increasing use in set-theoretic topology since it produces strong "Suslin-type" [18] consequences of MA $+\sim \mathrm{CH}$, e.g. all Aronszajn trees are special, compact spaces are hereditarily Lindelöf if and only if they are hereditarily separable, as well as - in the model we produce here - the important consequence of $V=L$ that all normal first countable spaces are collectionwise Hausdorff. These models are all obtained by starting with a model in which there is a coherent Suslin tree. This is a Suslin tree $S \subseteq \omega^{<\omega_{1}}$, closed under finite modifications, such that $\{\alpha \in \operatorname{dom}(s) \cap \operatorname{dom}(t): s(\alpha) \neq t(\alpha)\}$ is finite for all $s, t \in S$. The existence of such a tree follows from $\diamond[19$, 31] and holds after adding one Cohen real [36]. Once one has such an $S$, one then forces the maximal amount of some forcing axiom such as $\mathrm{MA}_{\omega_{1}}$ or PFA compatible with the existence of $S$. Then one 
forces with $S$. The details of how to do the penultimate forcing can be found in $[19,24,25$. Here we only need to know that these are iterations like those to establish $\mathrm{MA}_{\omega_{1}}$ or PFA, but that certain posets are omitted. For various propositions $\phi$, the proof that $\mathrm{MA}_{\omega_{1}}$ or PFA implies $\phi$ can be modified to prove that the weaker version of $\mathrm{MA}_{\omega_{1}}$ or PFA implies $S$ cannot force $\phi$ to fail. The $\phi$ in our case will comprise several propositions that together imply locally compact perfectly normal collectionwise Hausdorff spaces are paracompact. In addition, we either start from $L$ (if we do not require large cardinals so as to obtain as much of PFA as possible) or else a certain Easton model, and observe that the iteration plus the Suslin forcing will not destroy the fact that normal first countable $\aleph_{1}$-collectionwise Hausdorff spaces are collectionwise Hausdorff. The final step is to show that forcing with $S$ establishes that normal first countable spaces are $\aleph_{1}$-collectionwise Hausdorff. Consider then the following axioms:

$\mathrm{MA}_{\omega_{1}}(S)$ : There exists a coherent Suslin tree $S$, and if $P$ is a partial order satisfying the countable chain condition which does not force an uncountable antichain in $S$, and $D_{\xi}\left(\xi<\omega_{1}\right)$ is a sequence of dense open subsets of $P$, then there is a filter $G \subseteq P$ such that $G \cap D_{\xi} \neq \emptyset$ for each $\xi<\omega_{1}$.

$\operatorname{PFA}(S)$ : There exists a coherent Suslin tree $S$, and if $P$ is a proper partial order which does not force an uncountable antichain in $S$, and $D_{\xi}$ $\left(\xi<\omega_{1}\right)$ is a sequence of dense open subsets of $P$, then there is a filter $G \subseteq P$ such that $G \cap D_{\xi} \neq \emptyset$ for each $\xi<\omega_{1}$.

The consistency of $\mathrm{MA}_{\omega_{1}}(S)$ is established explicitly in [19], though very similar constructions had been studied earlier (in [9], for instance). The consistency of $\operatorname{PFA}(S)$ (minus the coherence requirement, which presents no additional difficulties), was established in [24].

We now introduce "PFA $(S)[S]$ implies $\phi$ " as an abbreviation for " $\phi$ holds whenever we force with $S$ over a model of $\operatorname{PFS}(S)$ ". We will say "there is a model of $\operatorname{PFA}(S)[S]$ in which $\phi$ holds" as an abbreviation for "there is a particular model of $\operatorname{PFA}(S)$ such that when we force with $S, \phi$ holds". We use analogous notation with $\mathrm{MA}_{\omega_{1}}$ in place of PFA.

Our key result is

Theorem 2. There is a model of $\operatorname{MA}_{\omega_{1}}(S)[S]$ and, if there is a supercompact cardinal, a model of $\operatorname{PFA}(S)[S]$, in which every normal first countable space is collectionwise Hausdorff.

We then get:

Theorem 3.

I. $\mathrm{MA}_{\omega_{1}}(S)[S]$ implies: 
(1) First countable hereditarily normal countable chain condition spaces are hereditarily separable.

II. $\operatorname{PFS}(S)[S]$ implies:

(2) Locally compact perfectly normal spaces of cardinality $\leq \aleph_{1}$ are metrizable.

III. Both models of Theorem 2 satisfy:

(3) All Whitehead groups are free.

IV. The $\operatorname{PFA}(S)[S]$ model of Theorem 2 satisfies:

(4) Locally compact perfectly normal spaces are paracompact.

(5) Every locally compact space with a hereditarily normal square is metrizable.

(6) Locally compact, locally hereditarily Lindelöf hereditarily normal spaces are paracompact if and only if they do not contain a perfect preimage of $\omega_{1}$.

(7) Hereditarily normal vector bundles are metrizable.

Recall that a space $X$ is countably tight if whenever $y \in \bar{Y} \subseteq X$, there is a countable $Z \subseteq Y$ such that $y \in \bar{Z}$. A subspace $Y$ of a space $X$ is locally countable if for each $y \in Y$ there is an open $U_{y}$ about $y$ containing only countably many members of $Y$. Furthermore, $Y$ is $\sigma$-discrete if it is the union of countably many discrete subspaces.

In addition to Theorem 2 our $\operatorname{PFA}(S)[S]$ results depend on the following unpublished theorem of Todorcevic:

THEOREM 4 ([37]). $\operatorname{PFA}(S)[S]$ implies that in a compact countably tight space, locally countable subspaces of size $\aleph_{1}$ are $\sigma$-discrete.

We shall also need:

LEMMA 5 ([21]). $\mathrm{MA}_{\omega_{1}}(S)[S]$ implies first countable hereditarily Lindelöf spaces are hereditarily separable.

Let us also mention for the benefit of the reader two other important unpublished applications of $\operatorname{PFA}(S)[S]$ due to Todorcevic.

THEOREM 6 ([39]). PFA $(S)[S]$ implies that hereditarily separable subspaces of compact countably tight spaces are hereditarily Lindelöf.

THEOREM 7 ([39]). PFA $(S)[S]$ implies every compact countably tight space is sequential.

We will not directly use these here. Theorem 6 is in fact an easy consequence of Theorem 4, while Theorem 7 is a crucial step toward establishing Theorem 4. 
In Section 1, we shall prove Theorem 2. In Section 2, we shall prove Theorem 1 and other topological consequences of Theorems 2 and 3. Section 3 provides an alternative, more direct, path to Theorem 1 for the readerperhaps a set theorist-who is not interested in more general results such as Theorem 3.IV.6 and does not want to wade through or depend on [1. In Section 4, we shall apply Theorem 2 to Whitehead groups. In Section 5, we shall talk about Theorem 4 and its current status.

1. Collectionwise Hausdorff. Let us recall some standard facts about "normality versus collectionwise normality" [33].

Definition. Let $\kappa$ be an infinite cardinal. A topological space is $\kappa$-collectionwise Hausdorff $(<\kappa$-collectionwise Hausdorff) if each closed discrete subspace $D$ of size $\leq \kappa(<\kappa)$ can be separated, i.e., there exist disjoint open sets $\left\{U_{d}\right\}_{d \in D}$ such that $d \in U_{d}$. A space is collectionwise Hausdorff if it is $\kappa$-collectionwise Hausdorff for every $\kappa$.

Definition ([11]). Let $\lambda$ be a regular uncountable cardinal. Then $\mathcal{A}=$ $\left\{A_{f}: f \in{ }^{\lambda} \lambda\right\}$ is a stationary system for $\lambda$ if each $A_{f}$ is a stationary subset of $\lambda$, and whenever $\alpha \in \lambda$ and $f, g \in{ }^{\lambda} \lambda$, if $f|\alpha=g| \alpha$ then

$$
A_{f} \cap(\alpha+1)=A_{g} \cap(\alpha+1) .
$$

$\diamond$ for stationary systems (at $\lambda$ ) is the assertion that for each stationary system $\mathcal{A}$ for $\lambda$, there is a sequence $\left\{f_{\alpha}\right\}_{\alpha<\lambda}$ such that $f_{\alpha} \in{ }^{\alpha} \alpha$ and for each $f \in{ }^{\lambda} \lambda$ there is a stationary $S \subseteq A_{f}$ such that $\beta \in S$ implies $f \mid \beta=f_{\beta}$.

Fleissner [1] proved:

Lemma 8. Suppose $\kappa$ is a regular uncountable cardinal, GCH holds at $\kappa$ and above, and $\diamond$ for stationary systems holds for all regular $\lambda \geq \kappa$. Then if $X$ is a normal first countable $<\kappa$-collectionwise Hausdorff space, then $X$ is collectionwise Hausdorff.

He also probably noticed the following results, but the only reference for them we know of is [34].

Lemma 9. Suppose $\lambda$ is a regular uncountable cardinal. Adjoin $\lambda^{+}$Cohen subsets of $\lambda$. Then $\diamond$ for stationary systems holds at $\lambda$.

Lemma 10. Suppose $\diamond$ for stationary systems holds at the regular uncountable cardinal $\lambda$. Force with a $\lambda$-chain condition partial order of size $\leq \lambda$. Then $\diamond$ for stationary systems still holds at $\lambda$.

Using these lemmas, it is not difficult to deduce that normal first countable spaces which are $\aleph_{1}$-collectionwise Hausdorff will be collectionwise Hausdorff in the model obtained by forcing with $S$ over a model of $\operatorname{PFA}(S)$, provided we start with an appropriate model over which to do the $\operatorname{PFA}(S)$ 
iteration. In particular, start with a model in which there is a supercompact cardinal $\kappa$. To simplify matters, we could establish GCH below $\kappa$ by a "mild" forcing [15] keeping $\kappa$ supercompact. We then make $\kappa$ indestructible under $\kappa$-directed-closed forcing [22] and then Easton-force to add $\lambda^{+}$Cohen subsets of $\lambda$ for every regular cardinal $\lambda \geq \kappa$ [5]. This will establish $\diamond$ for stationary systems for regular $\lambda \geq \kappa$, while keeping $\kappa$ supercompact. We then force to create a coherent Suslin tree $S$, then force $\operatorname{PFA}(S)$ and lastly force with $S$. The iteration of these three forcings has the $\kappa$-chain condition and is of size $\kappa$, so we have established that normal first countable spaces that are $<\kappa$-collectionwise Hausdorff are collectionwise Hausdorff. It is clear that the straightforward iteration to produce $\operatorname{PFA}(S)$-if it works at all-will produce a model in which $\kappa=\aleph_{2}$, but in fact Farah [9] proves $\operatorname{PFA}(S)$ implies OCA, while $\operatorname{PFA}(S)$ implies $\mathrm{MA}(\sigma$-centred) because $\sigma$-centred forcing does not add uncountable chains to Suslin trees [18] (see also [19]). It follows (see [3]) that

Lemma 11. $\operatorname{PFA}(S)$ implies that $2^{\aleph_{0}}=\aleph_{2}$ and therefore so does $\operatorname{PFA}(S)[S]$.

The second part of Lemma 11 follows from the fact that forcing with a Suslin tree preserves cardinals and does not add reals. This gives the following lemma.

LEMMA 12. Let $\kappa$ be a supercompact cardinal, and assume that $\diamond$ for stationary systems holds for every regular cardinal $\lambda \geq \kappa$. In the model obtained by first forcing $\operatorname{PFA}(S)$ by a $\kappa$-c.c. forcing of size $\kappa$ and then forcing with $S$, normal first countable $\aleph_{1}$-collectionwise Hausdorff spaces are collectionwise Hausdorff.

A simplified version of the above remarks and Lemma 12 applies to $\operatorname{MA}_{\omega_{1}}(S)[S]$. We can start with the Easton forcing, or simply with $V=L$. In either case $\diamond$ for stationary systems holds at regular cardinals and there is a coherent Suslin tree $S$. We then force with c.c.c. partial orders preserving $S$ to establish $\mathrm{MA}_{\omega_{1}}(S)$ and $2^{\aleph_{0}}=\aleph_{2}$, and then force with $S$. As before, in this model of $\mathrm{MA}_{\omega_{1}}(S)[S]$, normal first countable $\aleph_{1}$-collectionwise Hausdorff spaces are collectionwise Hausdorff.

It remains to prove normal first countable spaces are $\aleph_{1}$-collectionwise Hausdorff in these models. In order to do that, we prove a purely settheoretic combinatorial lemma:

Lemma 13. After forcing with a Suslin tree, the following holds. Suppose that $\left\{N(\alpha, i): i<\omega, \alpha<\omega_{1}\right\}$ are sets such that for all $\alpha, i, N(\alpha, i+1) \subseteq$ $N(\alpha, i)$. Suppose further that for all $A \subseteq \omega_{1}$, there is an $f: \omega_{1} \rightarrow \omega$ such that

$$
\bigcup\{N(\alpha, f(\alpha)): \alpha \in A\} \cap \bigcup\left\{N(\beta, f(\beta)): \beta \in \omega_{1} \backslash A\right\}=\emptyset .
$$


Then there is a $g: \omega_{1} \rightarrow \omega$ and a closed unbounded $C \subseteq \omega_{1}$ such that whenever $\alpha<\beta$ and $C \cap(\alpha, \beta] \neq \emptyset$, then $N(\alpha, g(\alpha)) \cap N(\beta, g(\beta))=\emptyset$.

COROllary 14. In any model obtained by forcing with a Suslin tree, in particular, in any model of $\mathrm{MA}_{\omega_{1}}(S)[S]$ or $\mathrm{PFA}(S)[S]$, normal first countable spaces are $\aleph_{1}$-collectionwise Hausdorff.

Proof. Without loss of generality we may assume that the topology is on a member of $V$; let the $N(\alpha, i)$ 's be a descending neighborhood base at $\alpha$, where we have labeled the points of a discrete closed subspace of the space $X$ with the countable ordinals. Let $g$ and $C$ be as in the statement of Lemma 13 . Define $c: \omega_{1} \rightarrow \omega_{1}$ by letting $c(\alpha)=\sup ((C-\{0\}) \cap(\alpha+1))$, and let $\alpha \sim \beta$ if $c(\alpha)=c(\beta)$. The $\sim$-classes are countable and normality implies $\aleph_{0^{-}}$ collectionwise Hausdorffness, so there is a $q: \omega_{1} \rightarrow \omega$ such that $c(\alpha)=c(\beta)$ implies $N(\alpha, q(\alpha)) \cap N(\beta, q(\beta))=\emptyset$. Let $r(\alpha)=\max (g(\alpha), q(\alpha))$. Then $\{N(\alpha, r(\alpha))\}_{\alpha<\omega_{1}}$ is the required separation.

Proof of Lemma 13. Let $S$ be a Suslin tree. Let $\left\{\dot{N}(\alpha, i): i<\omega, \alpha<\omega_{1}\right.$, $\left.\alpha<\omega_{1}\right\}$ be $S$-names for subsets of $X$ as in the hypothesis. For $s \in S$, let $\ell(s)$ be the length of $s$. Since $S$ has countable levels and its corresponding forcing poset is $\omega$-distributive, we can construct an increasing function $h: \omega_{1} \rightarrow \omega_{1}$ such that

for all $\alpha<\omega_{1}$ and all $s \in S$ with $\ell(s)=h(\alpha), s$ decides all statements of the form " $\dot{N}(\beta, j) \cap \dot{N}(\alpha, i)=\emptyset$ ", for all $i, j<\omega$ and $\beta<\alpha$.

Let $\dot{A}$ be an $S$-name for a subset of $\omega_{1}$ such that for no $\alpha<\omega_{1}$ does any $s \in S$ with $\ell(s)=h(\alpha)$ decide whether $\alpha \in A$. To define such an $\dot{A}$, for each $\alpha<\omega_{1}$ divide the immediate successors of each $s \in S$ with $\ell(s)=h(\alpha)$ into two non-empty sets: let the members of one set force $\alpha \in \dot{A}$ and let the members of the other set force $\alpha \notin \dot{A}$.

Let $\dot{f}$ be an $S$-name for a function $f: \omega_{1} \rightarrow \omega$ as in the hypothesis of the lemma, with respect to $A$. Let $C$ be a closed unbounded subset of $\omega_{1}$ in $V$ such that for each $s \in S$ with $\ell(s) \in C, s$ decides $f \mid \ell(s)$ and $A \mid \ell(s)$, and such that for all $\alpha<\beta<\omega_{1}$, if $\beta \in C$ then $h(\alpha)<\beta$. We will define an $S$-name $\dot{g}$ for a function from $\omega_{1}$ to $\omega$ such that whenever $\alpha<\beta<\omega_{1}$,

$$
\text { if }(\alpha, \beta] \cap C \neq \emptyset \text {, then } N(\alpha, g(\alpha)) \cap N(\beta, g(\beta))=\emptyset \text {. }
$$

Let $c: \omega_{1} \rightarrow \omega_{1}$ be defined by $c(\beta)=\sup (C \cap(\beta+1))$. Then for all $\beta<\alpha<\omega_{1}, c(\alpha)=c(\beta)$ if and only if $C \cap(\beta, \alpha]=\emptyset$. Fix $\beta<\omega_{1}$. Each $s \in S$ with $\ell(s)=h(\beta)$ decides $f \mid c(\beta)$ and $A \mid c(\beta)$ and " $\dot{N}(\alpha, f(\alpha)) \cap \dot{N}(\beta, i)=\emptyset$ " for all $i<\omega, \alpha<c(\beta)$, but not whether $\beta \in A$. Fix $s \in S$ with $\ell(s)=h(\beta)$. Since $s$ does not decide whether $\beta \in A$, we claim that there is an $i_{0}<\omega$ such that

for all $\alpha<c(\beta)$ such that $s \Vdash \alpha \in \dot{A}, s \Vdash \dot{N}(\alpha, \dot{f}(\alpha)) \cap \dot{N}\left(\beta, i_{0}\right)=\emptyset$. 
To see this, extend $s$ to $t \in S$ forcing that $\beta \notin A$ and deciding $f(\beta)$. Let $i_{0}$ be the value of $f(\beta)$ as decided by $t$. Then for each $\alpha<c(\beta)$ such that $s \Vdash \alpha \in \dot{A}$, $t$ forces that $N(\alpha, f(\alpha)) \cap N\left(\beta, i_{0}\right)=\emptyset$, but these facts were already decided by $s$. Similarly, there is an $i_{1}<\omega$ such that

$$
\text { for all } \alpha<c(\beta) \text { such that } s \Vdash \alpha \notin \dot{A}, s \Vdash \dot{N}(\alpha, \dot{f}(\alpha)) \cap \dot{N}\left(\beta, \dot{i}_{1}\right)=\emptyset \text {. }
$$

Since $s$ decides $A \mid c(\beta)$, letting $\bar{i}=\max \left\{i_{0}, i_{1}\right\}$,

$$
\text { for all } \alpha<c(\beta), s \Vdash \dot{N}(\alpha, \dot{f}(\alpha)) \cap \dot{N}(\beta, \bar{i})=\emptyset .
$$

We have such an $\bar{i}_{s}$ for each $s$ in the $h(\beta)$ th level of the tree, so we can construct a name $\dot{g}$ such that

$$
s \Vdash \dot{g}(\beta)=\max \left\{\bar{i}_{s}, \dot{f}(\beta)\right\}
$$

for each $s \in S$ with $\ell(s)=h(\beta)$. Then $\dot{g}$ is as required.

2. Topological applications. The compatibility of "locally countable subspaces of size $\aleph_{1}$ in a compact countably tight space are $\sigma$-discrete" with "normal first countable spaces are collectionwise Hausdorff" enables us to strengthen a variety of results of Balogh [1] and other authors, in particular proving Theorem 1, which we shall soon establish.

Let us first prove (1) of Theorem 3. We know that normal first countable spaces are $\aleph_{1}$-collectionwise Hausdorff and that first countably hereditarily Lindelöf spaces are hereditarily separable. Let $X$ be a hereditarily normal first countable countable chain condition space. Let $Y \subseteq X$. Since $X$ is hereditarily collectionwise Hausdorff, $Y$ cannot have an uncountable discrete subspace. It is standard that $Y$ must therefore have a dense hereditarily Lindelöf subspace. For recursively define $x_{\alpha}$ such that $x_{\alpha} \notin \overline{\left\{x_{\beta}: \beta<\alpha\right\}}$, until for some $\lambda,\left\{x_{\alpha}: \alpha<\lambda\right\}$ is dense. We claim $\left\{x_{\alpha}: \alpha<\lambda\right\}$ is hereditarily Lindelöf. For if not, there would exist $\left\{x_{\alpha_{\gamma}}: \gamma<\omega_{1}\right\}$ such that $\left\{x_{\alpha_{\gamma}}: \gamma<\delta\right\}$ is open in $\left\{x_{\alpha_{\gamma}}: \gamma<\omega_{1}\right\}$, for each $\delta<\omega_{1}$. But then $\left\{x_{\alpha_{\gamma}}: \gamma<\omega_{1}\right\}$ is discrete. Thus, since $\left\{x_{\alpha}: \alpha<\lambda\right\}$ is dense and hereditarily Lindelöf, by Lemma $5, Y$ is separable.

Next, we will need some facts due to Balogh.

Definition. $f: X \rightarrow Y$ is perfect if it is continuous, closed, and inverse images of points are compact.

The same argument that proves that the set of limit ordinals in $\omega_{1}$ is not a $G_{\delta}$ extends to show that:

Lemma 15 ([1]). A perfectly normal space does not include a perfect preimage of $\omega_{1}$.

We also have: 
Lemma 16 (1]). If $X$ is locally compact and countably tight, then the one-point compactification of $X$ is countably tight if and only if $X$ does not include a perfect preimage of $\omega_{1}$.

Balogh [1] proved under MA $+\sim \mathrm{CH}$ that connected, locally compact, locally hereditarily Lindelöf, hereditarily normal collectionwise Hausdorff spaces are paracompact if and only if they do not include a perfect preimage of $\omega_{1}$. We drop two of these conditions and get:

Theorem 17. In the $\mathrm{PFA}(S)[S]$ model of Theorem 2, locally compact, locally hereditarily Lindelöf, hereditarily normal spaces are paracompact if and only if they do not include a perfect preimage of $\omega_{1}$.

Theorem 1 follows easily. Theorem 17 answers a question Balogh asked for manifolds in [1]. Theorem 17 will follow immediately from the following lemma, which is essentially due to Balogh [1] (see the proof of his Theorem 3.3).

Lemma 18. Suppose locally countable subspaces of size $\aleph_{1}$ of a compact countably tight space are $\sigma$-discrete. If $X$ is locally hereditarily Lindelöf, locally hereditarily separable, hereditarily collectionwise Hausdorff, and can be embedded into a countably tight compact space, then $X$ is paracompact.

Proof of Theorem 17. Let $X$ satisfy the conditions of Theorem 17 . We claim it is paracompact. We know that the one-point compactification of $X$ is countably tight, that $X$ is first countable, and hence that $X$ is hereditarily collectionwise Hausdorff. By Lemma $5, X$ is locally hereditarily separable, so Lemma 18 applies. On the other hand, if $X$ is paracompact, it cannot include a closed perfect preimage of $\omega_{1}$, but such preimages are closed in first countable spaces.

TheOREM 19. PFA $(S)[S]$ implies every locally compact perfectly normal space of cardinality $\aleph_{1}$ is metrizable.

Proof. We first note that a space such as in the theorem has a countable neighborhood around each point. This follows from Lemma 11 and the fact that compact first countable spaces have cardinality either $\aleph_{0}$ or $2^{\aleph_{0}}$. But countable compact spaces are metrizable and paracompact locally metrizable spaces are metrizable, so we need only establish that the space is paracompact. Due to the size restriction, $\aleph_{1}$-collectionwise Hausdorffness will suffice.

As a corollary of Theorem 17, we will get a metrization theorem which answers a question in [2]:

Definition. $X$ has a $G_{\delta}$-diagonal if $\{\langle x, y\rangle: x=y\}$ is a $G_{\delta}$ in $X \times X$. 
Lemma 20 ([4]). A countably compact space with a $G_{\delta}$-diagonal is metrizable (and hence compact).

Theorem 21. In the $\operatorname{PFA}(S)[S]$ model of Theorem 2, every locally compact hereditarily normal space with a $G_{\delta}$-diagonal is metrizable.

Proof. It suffices to show $X$ is paracompact, since it is locally metrizable. It follows from Lemma 20 that $X$ does not include a perfect preimage of $\omega_{1}$. Clearly $X$ is first countable, locally hereditarily Lindelöf, and locally hereditarily separable. But then Lemma 18 applies.

It was shown in [21] that in the extension produced by forcing with the Suslin tree $S$ over a model of $\mathrm{MA}_{\omega_{1}}(S)$, every compact space with hereditarily normal square is metrizable. We shall extend this to locally compact spaces by using the PFA $(S)[S]$ model considered in this paper, thus obtaining (5) of Theorem 3 ,

Again, we suspect that the supercompact cardinal is not necessary, though we think it unlikely that the locally compact result can be obtained from the compact case for the following reason. Katětov [16] proved that every compact space with hereditarily normal cube is metrizable. There is no such ZFC result for locally compact spaces - it is routine to show:

ThEOREM 22. $\mathrm{MA}_{\omega_{1}}$ implies there is a locally compact non-metrizable space $X$ with $X^{n}$ hereditarily normal for all $n \in \omega$.

Proof. This is standard. $X$ will be any subset of the real line of size $\aleph_{1}$ with the following topology. Let $D$ be countable dense in $X$ in the real line subspace topology. We make each point of $D$ isolated. For each $x \in X-D$, we fix a sequence from $D$ converging to $x$, and let a neighborhood of $x$ be $\{x\}$ together with a tail of the sequence. Then $X$ is locally compact and non-metrizable, as is $X^{n}$ for each $n \in \omega$. Now, $X^{n}$ has a weaker separable metrizable topology, as a subspace of $\mathbb{R}^{n}$. Each point of $X^{n}$ has a neighborhood base consisting of sets which are compact in that weaker topology. By the following lemma, $\mathrm{MA}_{\omega_{1}}$ will imply $X^{n}$ is hereditarily normal.

Lemma 23 ([43, Section 7.1]). Assume MA $(\sigma$-centred $)+\sim \mathrm{CH}$. Suppose $\rho$ and $\tau$ are two topologies on a set $X$ such that

(i) $\rho \subseteq \tau$,

(ii) $\langle X, \rho\rangle$ is Hausdorff and second countable,

(iii) there is a closed neighborhood base for $\tau$ consisting of sets compact in $\langle X, \rho\rangle$.

Then for all $H, K \in[X]^{<2^{\aleph_{0}}}$ such that $\bar{H} \cap K=H \cap \bar{K}=\emptyset$ in the $\tau$ topology, we have disjoint open $U_{H}$ and $U_{K}$ in $\tau$ including $H$ and $K$ respectively. 
Now to prove (5) of Theorem 3, we will need a lemma of Katětov [16]:

LEMma 24. If $Y$ is countably compact and $Y^{2}$ is hereditarily normal, then $Y$ is perfectly normal.

Now suppose that $X$ is locally compact and $X^{2}$ is hereditarily normal. By [21] it follows that $X$ is locally metrizable and hence locally hereditarily Lindelöf. $X$ is homeomorphic to a subspace of $X^{2}$, so it too is hereditarily normal. Since $X$ is locally metrizable, to show it is metrizable it suffices to show it is paracompact. This will follow from Theorem 17 if we can show $X$ includes no perfect preimage of $\omega_{1}$. Suppose it had such a preimage $Y$. Then $Y$ would be countably compact and, by Katětov's lemma, perfectly normal. But that is impossible by Lemma 15.

Another consequence of the approach taken in this paper is the following result.

Theorem 25. In the $\operatorname{PFA}(S)[S]$ model of Theorem 2, every hereditarily normal vector bundle is metrizable.

Compare this with Nyikos' $\mathrm{MA}_{\omega_{1}}$ implies every hereditarily collectionwise Hausdorff vector bundle is metrizable [26]. More on vector bundles, including their definition, can be found in [32, pp. 245-257].

Following Nyikos [27], we have:

Definition. A space $X$ is of Type $I$ if $X=\bigcup_{\alpha<\omega_{1}} U_{\alpha}$, where the $U_{\alpha}$ 's are open, $\bar{U}_{\beta} \subseteq U_{\alpha}$ whenever $\beta<\alpha, U_{\alpha}=\bigcup_{\beta<\alpha} U_{\beta}$ for limit $\alpha$, and each $\bar{U}_{\alpha}$ is Lindelöf.

Proof of Theorem 25. Vector bundles are connected manifolds, so by Theorem 17, it suffices to show that hereditarily normal ones do not include perfect preimages of $\omega_{1}$. Nyikos [26] proved that vector bundles of Type I cannot include a perfect preimage of $\omega_{1}$, so it suffices to show that hereditarily normal vector bundles are of Type I.

Manifolds are first countable, so our vector bundle $V$ is hereditarily collectionwise Hausdorff. Hereditarily collectionwise Hausdorff connected manifolds have Lindelöf degree $\leq \aleph_{1}$ by [1, Lemma 3.2]. It follows that $V=\bigcup_{\alpha<\omega_{1}} U_{\alpha}$, where each $U_{\alpha}$ is hereditarily Lindelöf. Let $V_{\alpha}=\bigcup_{\beta<\alpha} U_{\beta}$. Then $V$ will be Type I if each $\bar{V}_{\alpha}$ is Lindelöf. Since $V_{\alpha}$ has no uncountable discrete subspace, and $\bar{V}_{\alpha}$ is hereditarily collectionwise Hausdorff, $\bar{V}_{\alpha}$ as well as its one-point compactification $\bar{V}_{\alpha}^{*}$ also do not have uncountable discrete subspaces. Then $\bar{V}_{\alpha}^{*}$ is countably tight, so by Theorem 4 , any rightseparated subspace of size $\aleph_{1}$ would be $\sigma$-discrete. Thus $\bar{V}_{\alpha}^{*}$, and hence $\bar{V}_{\alpha}$, is hereditarily Lindelöf.

A more interesting question concerns the metrizability of hereditarily normal manifolds of dimension greater than 1. Nyikos has written several 
papers on the subject, proving for example from the consistency of a supercompact cardinal that such manifolds are metrizable if in addition they are hereditarily collectionwise Hausdorff [28]. We make the following conjecture:

CONJECTURE. If it is consistent that there is a supercompact cardinal, then it is consistent that every hereditarily normal manifold of dimension greater than 1 is metrizable.

Furthermore, we expect that there is a proof of this conjecture using the approach taken in this paper. In unpublished work, the second author has shown that the conjecture could be established if one could prove that $\operatorname{PFA}(S)[S]$ implies every first countable perfect preimage of $\omega_{1}$ includes a copy of $\omega_{1}$. The conclusion is a consequence of PFA.

3. A different path to Theorem 1. We defined Type I spaces toward the end of the previous section. It is easy to see that if the $U_{\alpha}$ 's have empty boundaries closed unboundedly often, the space will be paracompact. Assume then that for a Type I perfectly normal space $X$, the boundaries of the $U_{\alpha}$ 's are non-empty for $\alpha$ in some stationary $S$. Pick $x_{\alpha} \in \overline{U_{\alpha}} \backslash U_{\alpha}$ for $\alpha \in S$. Then $\left\{x_{\alpha}: \alpha \in S\right\}$ will be locally countable and hence $\sigma$-discrete by Lemmas 15, 16 and Theorem 4, since compact perfectly normal spaces are first countable. Since perfectly normal spaces are hereditarily normal, $X$ will be hereditarily collectionwise Hausdorff. But then, pressing down on a stationary $S^{\prime} \subseteq S$ such that $\left\{x_{\alpha}: \alpha \in S^{\prime}\right\}$ is discrete, we get an uncountable disjoint collection of open sets in some $U_{\alpha}$, contradicting its hereditary Lindelöfness.

We have proved Theorem 1 for Type I spaces; to prove it in general, it suffices to prove the following lemma, which is a slight variation of [1, 3.2].

Lemma 26. Suppose $X$ is locally hereditarily Lindelöf, locally hereditarily separable, and hereditarily collectionwise Hausdorff. Then $X$ is the topological sum of clopen subspaces, each of Lindelöf degree $\leq \aleph_{1}$.

For then, by the argument at the end of the proof of Theorem 25 above, $X$ will be the sum of Type I spaces, and hence will be paracompact.

Proof of Lemma 26. Let $\mathcal{U}_{0}$ be a maximal disjoint collection of basic open sets in $X$. Suppose $\mathcal{U}_{\beta}, \beta<\alpha$, have been defined to be unions of countably many disjoint collections of basic open sets. Let

$$
F_{\alpha}=X-\bigcup_{\beta<\alpha} \bigcup \mathcal{U}_{\beta} .
$$

Let $\mathcal{V}_{\alpha}$ be a collection of basic open sets such that $\left\{F_{\alpha} \cap V: V \in \mathcal{V}_{\alpha}\right\}$ is a maximal disjoint collection of relatively open subsets of $F_{\alpha}$. Fix a dense countable subset $D_{V}$ in each $F_{\alpha} \cap V$. Any selection of points, one from each 
$D_{V}$, yields a discrete subset of $\bigcup \mathcal{V}_{\alpha}$, which may therefore be separated by basic open sets. We may therefore cover $\bigcup\left\{D_{V}: V \in \mathcal{V}_{\alpha}\right\}$ by a collection $\mathcal{U}_{\alpha}$ of basic open sets such that $\mathcal{U}_{\alpha}$ is the union of countably many collections of disjoint basic open sets. We claim that

$$
X=\bigcup_{\alpha<\omega_{1}} \bigcup \mathcal{U}_{\alpha}
$$

Indeed, suppose that this is false. Let $W$ be a basic open neighborhood of $x \in \bigcap_{\alpha<\omega_{1}} F_{\alpha}$. Since the $F_{\alpha}$ 's are descending and $W$ is hereditarily Lindelöf, there is an $\alpha<\omega_{1}$ such that $F_{\alpha} \cap W=F_{\alpha+1} \cap W$. Then $\bigcup \mathcal{U}_{\alpha+1} \cap W$ is empty, so $W \cap F_{\alpha+1}=\emptyset$. But that is a contradiction, as $x \in W \cap \bigcap_{\alpha<\omega_{1}} F_{\alpha}$.

Now $\bigcup_{\alpha<\omega_{1}} \mathcal{U}_{\alpha}$ is the union of $\aleph_{1}$ collections of disjoint open sets. Each member of $\bigcup_{\alpha<\omega_{1}} \mathcal{U}_{\alpha}$ meets only countably many elements of each such collection. Therefore $X$ is the sum of clopen subspaces, each composed of the union of $\aleph_{1}$ basic open sets.

4. Whitehead groups. Just as the question of when normality implies collectionwise normality led to many advances in set-theoretic topology, the question of when Whitehead groups are free has been similarly influential in set-theoretic algebra. For a short, accessible introduction to the subject, see [6]. For a comprehensive presentation, see [7]. All terms not defined here can be found in both references. Here we only want to point out:

Theorem 27. In the $\mathrm{MA}_{\omega_{1}}(S)[S]$ and $\mathrm{PFA}(S)[S]$ models discussed here, in which normal first countable spaces are collectionwise Hausdorff, all Whitehead groups are free.

Proof. We assume the reader is somewhat familiar with the proof that Whitehead groups are free in $L$. That proof proceeds by induction on the cardinality of the groups. It is true for countable ones, and for singular cardinals it is true if it is true for smaller cardinals, by a Singular Compactness Theorem.

At regular cardinals $\kappa$, for $S$ a stationary subset of $\kappa, \diamond(S)$ is sufficient to carry on with the induction. $\diamond$ for stationary systems is a stronger principle, so the case of $\kappa=\aleph_{1}$ is the only one needing consideration.

Shelah showed that there is a non-free Whitehead group of size $\aleph_{1}$ if and only if there is a ladder system on some stationary subset of $\omega_{1}$ which has the 2-uniformization property. But such a ladder system determines in a natural way a first countable, normal, non-collectionwise Hausdorff space of size $\aleph_{1}$.

It remains to be seen whether there are $\mathrm{MA}_{\omega_{1}}$ or PFA consequences holding in these models which would, in conjunction with "all Whitehead groups are free", produce results of algebraic interest. 
5. Remarks. According to Todorcevic (personal communication) the supercompact can probably be eliminated in the work of his on which Theorem 1 depends.

Most of this paper was written in 2003; it was submitted in 2009. The reason for the delay was that the authors had not seen the still unpublished proofs of Theorems 4 and 7.

At the 2006 Prague Topological Symposium, Todorcevic announced Theorem 4. He sketched the proof of Theorem 7 at the conference on Advances in Set-Theoretic Topology, in Honor of T. Nogura in Erice, Italy in 2008 [38.

Finite powers of compact countably tight spaces are countably tight [23]. Thus, in addition to Theorem 7 , one needs:

TheOREM 28 ([37]). $\operatorname{PFA}(S)[S]$ implies that if $K$ is a compact space with finite powers sequential, then every locally countable subset of $K$ of size $\aleph_{1}$ is $\sigma$-discrete.

At Todorcevic's suggestion, the details of Theorem 28 are supposed to appear in [10]. The proofs of Theorems 6 and 7 are supposed to appear in [39].

Acknowledgments. The authors acknowledge support from NSERC grant A-7354. The first author also acknowledges support from NSF-DMS0801009.

In conclusion, we thank the referee for many useful suggestions.

Note added in proof (October 8, 2010). We now can prove our conclusions from results of Todorcevic that do have accessible proofs. This is accomplished in the union of two preprints, one by each author, which prove a weaker version of Theorem 4, namely that the locally countable subspace is $\sigma$-discrete on a closed unbounded set.

\section{References}

[1] Z. T. Balogh, Locally nice spaces under Martin's axiom, Comment. Math. Univ. Carolin. 24 (1983), 63-87.

[2] Z. T. Balogh and H. Bennett, Conditions which imply metrizability in manifolds, Houston J. Math. 15 (1989), 153-162.

[3] M. Bekkali, Topics in Set Theory, in: Lecture Notes in Math. 1476, Springer, Berlin, 1991.

[4] J. Chaber, Conditions which imply compactness in countably compact spaces, Bull. Acad. Polon. Sci. Sér. Sci. Math. Astronom. Phys. 24 (1976), 993-998.

[5] W. B. Easton, Powers of regular cardinals, Ann. Math. Logic 1 (1970), 129-178.

[6] P. C. Eklof, Set theory generated by Abelian group theory, Bull. Symbolic Logic 3 (1997), 1-16.

[7] P. C. Eklof and A. H. Mekler, Almost Free Modules. Set-Theoretic Methods, NorthHolland, Amsterdam, 2002.

[8] R. Engelking, General Topology, Heldermann, Berlin, 1989. 
[9] I. Farah, $O C A$ and towers in $\mathcal{P}(\mathbb{N}) /$ fin, Comment. Math. Univ. Carolin. 37 (1996), 861-866.

[10] A. Fischer, Doctoral dissertation, University of Toronto, in preparation.

[11] W. G. Fleissner, Normal Moore spaces in the constructible universe, Proc. Amer. Math. Soc. 46 (1974), 294-298.

[12] G. Gruenhage, Paracompactness and subparacompactness in perfectly normal locally compact spaces, Russian Math. Surveys 35 (1980), no. 3, 49-55; Russian transl.: Uspekhi Mat. Nauk 35 (1980), no. 3, 44-49.

[13] R. E. Hodel, Cardinal functions, I, in: Handbook of Set-Theoretic Topology, K. Kunen and J. E. Vaughan (eds.), North-Holland, Amsterdam, 1984, 1-61.

[14] T. Jech, Set Theory, Springer, Berlin, 1997.

[15] A. Kanamori, The Higher Infinite, Springer, Berlin, 1994.

[16] M. Katětov, Complete normality of cartesian products, Fund. Math. 35 (1948), 271274.

[17] K. Kunen, Set Theory, North-Holland, Amsterdam, 1980.

[18] K. Kunen and F. D. Tall, Between Martin's Axiom and Souslin's Hypothesis, Fund. Math. 102 (1979), 173-181.

[19] P. Larson, An $\mathbb{S}_{\max }$ variation for one Souslin tree, J. Symbolic Logic 64 (1999), 81-98.

[20] P. Larson and S. Todorcevic, Chain conditions in maximal models, Fund. Math. 168 (2001), 77-104.

[21] —, - Katětov's problem, Trans. Amer. Math. Soc. 354 (2002), 1783-1791.

[22] R. Laver, Making the supercompactness of $\kappa$ indestructible under $\kappa$-directed closed forcing, Israel J. Math. 29 (1978), 385-388.

[23] V. I. Malyhin, On tightness and Suslin number in $\exp X$ and in a product of spaces, Dokl. Akad. Nauk SSSR 203 (1972), 1001-1003 (in Russian); English transl.: Soviet Math. Dokl. 13 (1972), 496-499.

[24] T. Miyamoto, $\omega_{1}$-Souslin trees under countable support iterations, Fund. Math. 142 (1993), 257-261.

[25] —, Iterating semiproper preorders, J. Symbolic Logic 67 (2002), 1431-1468.

[26] P. J. Nyikos, Set-theoretic topology of manifolds, in: General Topology and its Relations to Modern Analysis and Algebra V (Prague, 1981), Heldermann, Berlin, 1982, $513-526$.

[27] —, The theory of nonmetrizable manifolds, in: Handbook of Set-Theoretic Topology, K. Kunen and J. E. Vaughan (eds.), North-Holland, Amsterdam, 1984, 633-684.

[28] - Correction to: "Complete normality and metrization theory of manifolds" [Topology Appl. 123 (2002), 181-192], Topology Appl. 138 (2004), 325-327.

[29] A. Ostaszewski, On countably compact perfectly normal spaces, J. London Math. Soc. 14 (1976), 505-516.

[30] S. Shelah, Proper and Improper Forcing, Springer, Berlin, 1998.

[31] S. Shelah and J. Zapletal, Canonical models for $\aleph_{1}$-combinatorics, Ann. Pure Appl. Logic 98 (1999), 217-259.

[32] M. Spivak, Differential Geometry, Vol. 1, Publish or Perish, 1970.

[33] F. D. Tall, Normality versus collectionwise normality, in: Handbook of Set-Theoretic Topology, K. Kunen and J. E. Vaughan (eds.), North-Holland, Amsterdam, 1984, 685-732.

[34] - Covering and separation properties in the Easton model, Topology Appl. 28 (1988), 155-163.

[35] —, Tall's problems, in: Open Problems in Topology, J. van Mill and G. M. Reed (eds.), North-Holland, Amsterdam, 1990, 21-35. 
[36] S. Todorcevic, Partitioning pairs of countable ordinals, Acta Math. 159 (1987), 261294.

[37] —, lecture at International Topology Symposium in Prague, 2006.

[38] - Forcing with coherent Souslin tree, slides of lectures presented at Advances in Set-Theoretic Topology, in Honor of T. Nogura, Erice, Italy, 2008.

[39] - Chain conditions in topology, II, in preparation.

[40] S. Watson, Locally compact normal spaces in the constructible universe, Canad. J. Math. 34 (1982), 1091-1096.

[41] - Sixty questions on regular not paracompact spaces, in: Proc. 11th Winter School on Abstract Analysis (Železná Ruda, 1983), Rend. Circ. Mat. Palermo (2) Suppl. 1984, no. 3, 369-373.

[42] S. Watson, Problems I wish I could solve, in: Open Problems in Topology, J. van Mill and G. M. Reed (eds.), North-Holland, Amsterdam, 1990, 37-76.

[43] W. Weiss, Versions of Martin's Axiom, in: Handbook of Set-Theoretic Topology, K. Kunen and J. E. Vaughan (eds.), North-Holland, Amsterdam, 1984, 827-886.

Paul B. Larson

Department of Mathematics

Miami University

Oxford, OH 45056, U.S.A.

E-mail: larsonpb@muohio.edu
Franklin D. Tall

Department of Mathematics

University of Toronto Toronto, Ontario M5S 2E4, Canada

E-mail: tall@math.utoronto.ca

Received 21 August 2009;

in revised form 21 June 2010 